\title{
Portal Vein Thrombosis in a Preterm Newborn with Mutation of the MTHFR and PAl-1 Genes and Sepsis by Candida parapsilosis
}

\author{
Mario Giuffrè, MD, $\mathrm{PhD}^{1} \quad$ Clelia Lo Verso, MD $\quad$ Gregorio Serra, MD ${ }^{1}$ Giovanni Moceri, MD ${ }^{1}$ \\ Marcello Cimador, MD ${ }^{1}$ Giovanni Corsello, $\mathrm{MD}^{1}$ On behalf of the Study Group of Neonatal Infectious \\ Diseases Affiliated to the Italian Society of Neonatology
}

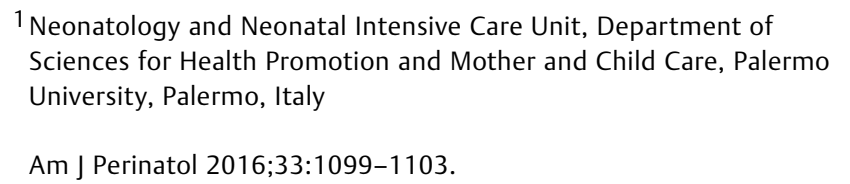

Address for correspondence Mario Giuffrè, MD, PhD, Neonatology and Neonatal Intensive Care Unit, Department of Sciences for Health Promotion and Mother and Child Care, Palermo University, via Alfonso Giordano 3, 90127 Palermo, Italy (e-mail: mario.giuffre@unipa.it).

\begin{abstract}
Objective This report discusses the role of both congenital and acquired risk factors in the pathogenesis of portal vein thrombosis (PVT).

Study Design We describe the clinical management and treatment of PVT in a preterm newborn with a homozygous mutation of the methylenetetrahydrofolate reductase (MTHFR) and plasminogen activator inhibitor-1 (PAl-1) genes and sepsis by Candida parapsilosis.

Results Although literature data suggest a minor role of genetic factors in thrombo-

Keywords

- portal thrombosis

- umbilical catheter

- fungal infection

- anticoagulation therapy

- thrombophilia

- gene polymorphism philia in the case of only one mutation, we hypothesize that combined thrombophilic genetic defects may have a cumulative effect and significantly increase the thrombotic risk.

Conclusion It could be appropriate to include more detailed analyses of procoagulant and fibrinolytic factors in the diagnostic workup of neonatal thrombosis, also through the investigation of genetic polymorphisms. The anticoagulant therapy and the removal of concurrent risk factors remain basic steps for the adequate management and prevention of complications.
\end{abstract}

Neonatal portal vein thrombosis (PVT) is a rare disease, with an incidence of 1 in 100,000 live births ${ }^{1}$ and 36 per 1,000 infants hospitalized in neonatal intensive care units (NICUs). ${ }^{2}$

The coagulation system of infants develops with age, as documented by considerable differences in the concentration of clotting proteins compared with adults; therefore, the thrombotic risk is significantly reduced in children. ${ }^{3}$

The etiology of portal vein occlusion in infants is multifactorial. Primitive (malformations, thrombophilic genetic defects, prematurity, low birth weight) and/or secondary (trauma, hypoxia) risk factors play a key role in the pathogenesis of the disease, which commonly occurs secondary to the placement of an umbilical vein catheter (UVC), with or without infection. ${ }^{4}$
Here, we report on a preterm newborn with PVT associated with mutation of the methylenetetrahydrofolate reductase (MTHFR) and plasminogen activator inhibitor-1 (PAI-1) genes and sepsis by Candida parapsilosis.

\section{Clinical Report}

A male neonate was born at $32+5$ gestational weeks in another NICU of town by cesarean delivery because of breech presentation. Intrauterine growth restriction (IUGR) occurred during pregnancy, likely due to extensively infarcted multiple hemorrhagic areas documented by histological examination of the placenta. Birth weight was 1,110 $\mathrm{g}$ (2nd centile), length ISSN 0735-1631. 
$37.5 \mathrm{~cm}$ (2nd centile), and head circumference $27.2 \mathrm{~cm}$ (2nd centile). Apgar scores measured at 1, 5 and 8 minutes were reported as 1,5 and 8 , respectively. The infant was immediately intubated and ventilated. A UVC was inserted and total parenteral nutrition (TPN) was started, integrated by minimal enteral feeding (MEF) with mother's milk administered through the nasogastric tube. On the sixth day of life, because of enteral feeding intolerance, MEF was suspended, the umbilical catheter was removed, and TPN was continued through a peripherally inserted central catheter (PICC). On the 11th day of life, enteral feeding with mother's milk and the formula was started again. After a week enteral nutrition was suspended again because of gastric residuals, abdominal distension, rigidity, and pain; abdominal X-rays revealed distension of ileum and not evident colon; laboratory tests revealed infection markers as thrombocytopenia (platelet count $34 \times 10^{3} / \mu \Lambda$ ), C-reactive protein $4.8 \mathrm{mg} / \mathrm{dL}$ (normal values $<1 \mathrm{mg} / \mathrm{dL}$ ), high levels of aminotransferases (aspartate aminotransferase: $732 \mathrm{U} / \mathrm{L}$, alanine aminotransferase: $234 \mathrm{U} / \mathrm{L}$ ), and signs of cholestasis (total bilirubin: $18.4 \mathrm{mg} / \mathrm{dL}$, direct bilirubin: $16.46 \mathrm{mg} / \mathrm{dL}$, gamma-glutamyl transferase: $137 \mathrm{U} / \mathrm{L}$, alkaline phosphatase: $578 \mathrm{U} / \mathrm{L}$ ). Blood culture was included in the diagnostic workup.

At 20 days of life, with the suspicion of intestinal occlusion, the baby was transferred to our NICU which is linked to a Pediatric Surgery Unit. On admission, he presented with jaundice, protruding abdomen, hepatosplenomegaly, bilateral hydrocele, hypotonia, and poor reactivity. Abdominal X-rays confirmed ileal obstruction and abdominal ultrasonography (US) showed diffused corpuscular effusion in the peritoneum, ileal loop distension with wall thickening. Patient parents received adequate communication and gave informed consent to the required surgical and medical treatments and the appropriate genetic investigations. Urgent surgical intervention of transverse laparotomy demonstrated the presence of a perihepatic abscess originated from a septic thrombus of the umbilical vein, drainage of the collection and removal of umbilical vessels were performed ( - Fig. 1 A, B). The day after admission, result of the blood culture performed before the transfer revealed Candida parapsilosis, therefore PICC was replaced and treatment with micafungin was started pending the results of the antimicogram, which subsequently showed susceptibility to the above-mentioned echinocandin (minimal inhibitory concentration [MIC] value: $2 \mu \mathrm{g} / \mathrm{mL}$ ), fluconazole and amphotericin-B (MIC value: $0.5 \mu \mathrm{g} / \mathrm{mL}$ for both). Postoperative abdominal US showed hepatosplenomegaly and a clot in the left branch of the hepatic portal vein (-Fig. 2) with subsequent extension to the right branch in the following week. Thrombophilic genetic analyses revealed a homozygous mutation in the MTHFR gene (C677T) and the homozygous 4G/4G polymorphism in the PAI-1 gene. Once excluded contraindications such as bleeding, intracranial hemorrhage, or cerebral infarct, treatment with subcutaneous enoxaparin was initiated at 1 month of life, with close instrumental monitoring of the thrombus and control of plasmatic levels of coagulation factors. The anticoagulant therapy was discontinued after 1 month of treatment because of reduction in the size of the clot and its regression to the left branch of the portal vein. No side effects have been reported.

During the first 3 weeks of hospitalization, general conditions of the patient were poor with persistently elevated inflammatory markers and worsening cholestasis. Repeated microbiological samples (blood cultures, septifast molecular test, and PICC culture) in the course of echinocandin treatment were still positive for C. parapsilosis and showed progressive decrease of susceptibility to micafungin until resistance. Therefore, fluconazole therapy (supported by the lowest MIC value, $0.06 \mu \mathrm{g} / \mathrm{mL}$ ) was added for a week. Finally, because of evidence of micafungin resistance and no clinical/biochemical improvement nor negativization of blood cultures, antifungal treatment was shifted to liposomal amphotericin-B (MIC value $0.5 \mu \mathrm{g} / \mathrm{mL}$ ) administered for 3 weeks, which allowed gradual clinical and biochemical

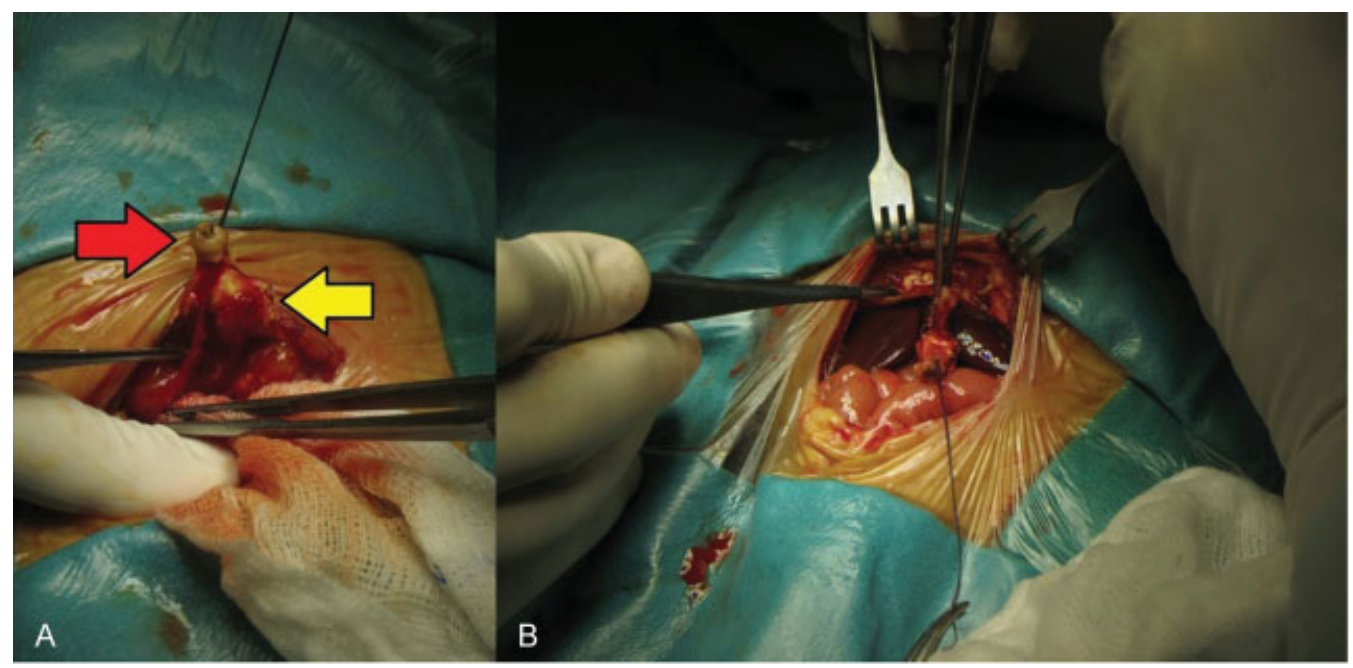

Fig. 1 (A) It is clearly visible the septic thrombus in the umbilical vein (upper arrow), and a small abscess in the hepatic inlet of the vessel (lower arrow); (B) extension of the perihepatic abscess to the falciform ligament and to the peritoneum. 


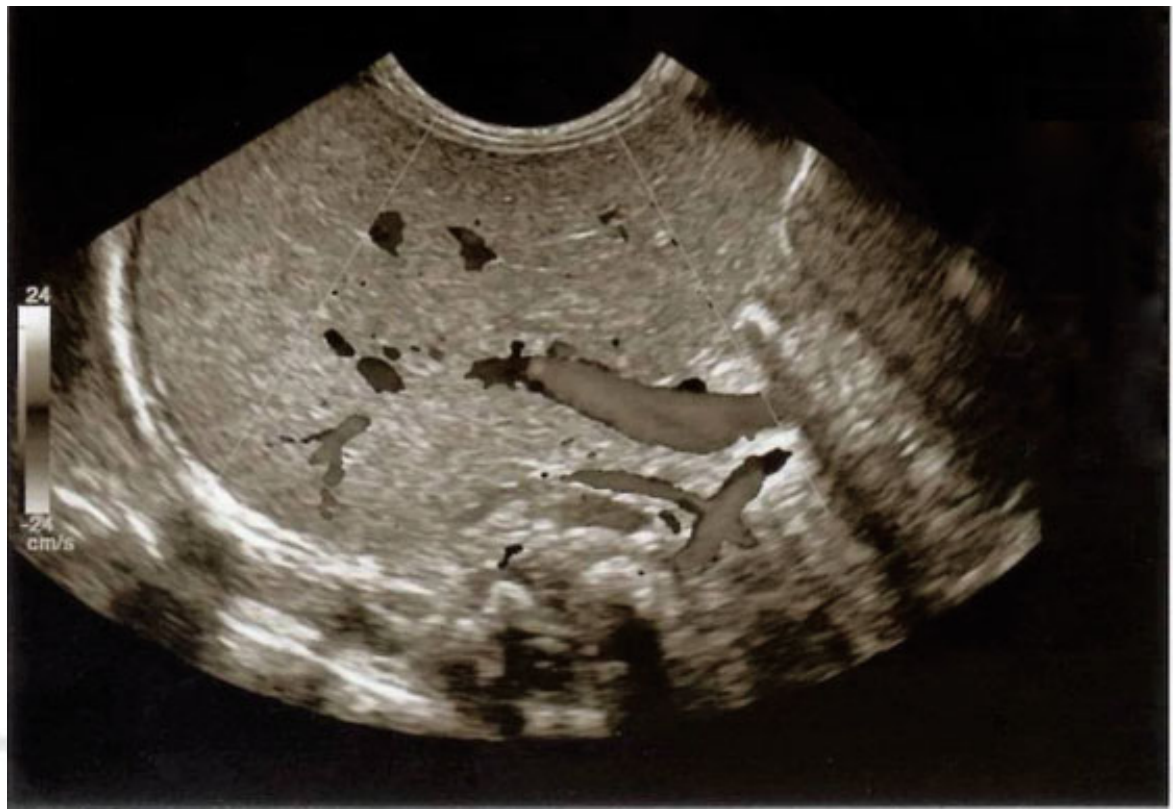

Fig. 2 Hepatic ultrasound shows the clot in the left branch of the hepatic portal vein.

improvement, reduction of the signs of cholestasis and of hypertransaminasemia, and negativization of molecular test and blood culture (after 10 and 20 days of treatment, respectively).

The infant was discharged in the 11th week of life in a good general health state and follow-up has been performed at Pediatric Surgery outpatient clinic in our department. At present, the patient is 18 months old, he develops normally, and liver US follow-up shows a partial regression of the thrombotic lesion with the improvement of portal vascular circulation.

\section{Discussion}

Neonatal PVT is a rare disease, with variable incidence from 0.7 to $5.1 / 100,000$ live births. ${ }^{5}$ Preterm newborns carry a significant risk for thrombosis, about half cases occurring in these patients, and continuous advances in medical and surgical management, which improve survival of preterm infants, may increase the incidence of thrombotic events. ${ }^{5}$

PVT is due both to congenital (malformations, thrombophilia, gestational age, birth weight, etc.) and secondary (infection, trauma, vessel catheterization, etc.) risk factors, with central venous line placement being the most important one in the neonatal period. In the case of improper insertion of the catheter into the portal vein, there may be thrombosis of hepatic vessels and other complications such as necrotizing enterocolitis, intestinal perforation, and portal hypertension. ${ }^{6}$ Perihepatic and liver abscesses are even rarer complications of a systemic infection in neonatal age. These uncommon events can happen, however, more easily in infants with sepsis and/or malposition of the umbilical catheter. Therefore, a diagnosis of hepatic/perihepatic abscess should be considered in any newborn with increasing markers of infection, thrombocytopenia, and abdominal manifestations (feeding intolerance, protrud- ing abdomen, hepatosplenomegaly). All these signs were present in our patient, although we cannot establish with reasonable certainty if the thrombus occurred before or after the fungal infection, since both are plausible causes of the clinical symptoms.

Two studies reported that low birth weight and early gestational age (especially with IUGR) could predispose to thrombosis, as occurred in our patient. ${ }^{7}$ Moreover, there have been reports of thrombophilia associated with PVT in newborns. ${ }^{4}$ However, the contribution of genetic or acquired thrombophilia in the causation of thrombosis in preterm infants is unknown. It is likely that congenital and acquired risk factors are present in the same patient. A condition of inherited thrombophilia should be suspected and screened in the case of recurrent thromboembolism, early onset in young age, unusual localization (mesenteric veins, portal vein, cerebral veins), and familiarity. There are no global tests, to date, that allow an easy diagnosis of thrombophilia, but there is the need to perform several analyses according to a standardized protocol. ${ }^{8}$ Among candidate genes for thrombotic risk, there are mainly those encoding for procoagulant factors including factor V, factor XIII, human platelet alloantigens, and $\beta$-fibrinogen. Some polymorphisms of the fibrinolytic system (PAI-1), homocysteine cycle (MTHFR), renin-angiotensin system $(A C E)$, and of lipid metabolism (APO-B and $A P O-E)$ are positively or negatively associated with thrombosis. We studied all these genes in our patient and we found two mutations/polymorphisms. The MTHFR gene carries a homozygous C677T mutation, which determines a substitution of alanine with valine in the final protein with consequent decreased enzymatic activity, very high levels of homocysteine and severe thrombotic events. In addition, we found a homozygous one base pair polymorphism $4 \mathrm{G} / 4 \mathrm{G}$ in the promoter region of $P A I-1$ gene, which consists in a deletion of a guanine at position 675 compared with the wild type $5 \mathrm{G}$. 
The 4G allele could just bind enhancers of transcription, while $5 \mathrm{G}$ interacts with enhancers and suppressors; this results in a lower level of transcription in the presence of the $5 \mathrm{G}$ allele. Several studies demonstrated that 4G/5G individuals have higher PAI-1 plasmatic levels with consequent inhibition of fibrinolysis and stored fibrin; homozygotes 4G/4G have PAI-1 plasmatic levels 25\% higher than 5G/5G subjects, with increased thrombotic risk. ${ }^{8}$ Thus, although some reports did not find an association between thrombophilia and thrombosis and MTHFR did not appear to add significant risk for neonatal PVT, ${ }^{4}$ preterm infants with prothrombotic mutations are more likely to develop thrombotic events in case of concurrent clinical triggers such as sepsis or indwelling catheters. Moreover, the unique genetic pattern of our patient with a double homozygous mutation/polymorphism of the MTHFR and PAI-1 genes, not described to date, could have significantly increased the thrombotic risk. This hypothesis is also strengthened by the placental histological findings of fetal thrombotic vasculopathy (FTV), that was likely responsible for the IUGR. FTV together with a history of recurrent miscarriages were highly suggestive for inherited thrombophilia. Genetic analyses of parental thrombophilic profile revealed they were both heterozygous for the same MTHFR mutation of the propositus, in addition, the father resulted homozygous and the mother heterozygous for the $4 \mathrm{G}$ polymorphism of PAI-1. These results were compatible with the homozygous condition of the proband for both the genes.

The long permanence ( 6 days) of the UVC may have contributed increasing the chance of complications together with the other risk factors. According to literature data, 4,6,7,9 we agree on the importance of limiting the length of stay of central vein catheters in neonates (72-96 hours for UVC).

C. parapsilosis sepsis certainly influenced the clinical course, acting as a concurrent trigger (or even an additional risk factor) for the thrombus, and was finally eradicated by liposomal amphotericin-B treatment. Although the benefit of azole prophylaxis in reducing the incidence of invasive candidiasis in small infants is well documented and micafungin has a specific authorization (license) for neonatal treatment, ${ }^{10}$ concerns have been raised over both fluconazole and micafungin efficacy in vivo. The lowest MIC values were registered for fluconazole, but we did not observed any clinical benefit likely representing an inability to act in a specific fungal sanctuarium (such as the thrombus or the indwelling catheter biofilm) rather than a sign of emerging azole resistance. In the past decade, echinocandins are proving to be a very useful addition to the antifungal armamentarium and have shown activity against the biofilms associated with various Candida species, including C. parapsilosis. ${ }^{11}$ Nevertheless, micafungin treatment was not successful in our patient and the progressive increase of MIC values up to resistance may reflect, in our experience, a lower efficacy against $C$. parapsilosis (as previously documented by other authors ${ }^{12,13}$ ) rather than a mechanical barrier by the thrombus which obstacles the antifungal activity on the cell wall, causing then second the establishment of resistance. Furthermore, although worldwide surveillance of $C$. parapsilosis indicated very low rates of resistance and low inci- dence of nonwild type, it cannot be excluded that mutation or acquisition of genes not known to date could change its resistance profile and then the epidemiology of the infection. Therefore, ongoing monitoring appears to be necessary to determine if strains of $C$. parapsilosis and other Candida spp. are developing resistance to fluconazole and/or to echinocandins. ${ }^{12}$ In this perspective whole fungal genome sequencing could be useful, to detect new possible mutations able to induce resistance and, as a result, to promptly address the most appropriate antifungal treatment.

Low-molecular-weight heparin or enoxaparin is the drug of first choice for anticoagulant therapy in the neonatal period, because of its more stable pharmacokinetics, ease of administration and less frequent monitoring. ${ }^{5}$ The optimal duration of anticoagulant therapy in newborns is not yet well defined; however, it is generally recommended a short treatment period (from 10 days to 3 months) with regular monitoring of the thrombus. ${ }^{14}$

Enoxaparin treatment for a month proved to be effective in our patient together with appropriate antifungal treatment, avoiding the extension of the thrombus and allowing its partial resolution at 1 year follow-up. If the PVT does not resolve spontaneously and/or it is not treated, the patient may develop complications (cavernoma, portal hypertension, liver lobe atrophy). ${ }^{4}$

Although literature data suggest a minor role of genetic factors in thrombophilia in the case of only one mutation, ${ }^{4}$ the coexistence of two homozygous mutations/polymorphisms in our patient seems to have significantly increased the thrombotic risk. Therefore, it seems useful to include in the diagnostic workup of neonatal thrombosis not only the investigation of procoagulant factors, but also the study of the fibrinolytic system. However, the removal/resolution of eventual additional concurrent risk factors (UVC, fungal infection) and the anticoagulant therapy are necessary steps to address a favorable clinical course and prevent further complications.

\section{Conflict of Interest \\ None.}

\section{References}

1 Heller C, Schobess R, Kurnik K, et al; For the Childhood Thrombophilia Study Group. Abdominal venous thrombosis in neonates and infants: role of prothrombotic risk factors - a multicentre casecontrol study. Br J Haematol 2000;111(2):534-539

2 Morag I, Epelman M, Daneman A, et al. Portal vein thrombosis in the neonate: risk factors, course, and outcome. J Pediatr 2006; 148(6):735-739

3 Nowak-Göttl U, Janssen V, Manner D, Kenet G. Venous thromboembolism in neonates and children-update 2013. Thromb Res 2013;131(Suppl 1):S39-S41

4 Williams S, Chan AK. Neonatal portal vein thrombosis: diagnosis and management. Semin Fetal Neonatal Med 2011;16(6): 329-339

5 Bhat R, Monagle P. The preterm infant with thrombosis. Arch Dis Child Fetal Neonatal Ed 2012;97(6):F423-F428 
6 Bothur-Nowacka J, Czech-Kowalska J, Gruszfeld D, et al. Complications of umbilical vein catherisation. Case Report. Pol J Radiol 2011;76(3):70-73

7 Park CK, Paes BA, Nagel K, Chan AK, Murthy P; Thrombosis and Hemostasis in Newborns (THiN) Group. Neonatal central venous catheter thrombosis: diagnosis, management and outcome. Blood Coagul Fibrinolysis 2014;25(2):97-106

8 Ferraù $\mathrm{V}$, Ferro E, Barone $\mathrm{C}$, et al. Thrombophilia screening: clinical and genetic aspects. Ital J Genet Pediatr Immunol 2011;1:3

9 Yumani DF, van den Dungen FA, van Weissenbruch MM. Incidence and risk factors for catheter-associated bloodstream infections in neonatal intensive care. Acta Paediatr 2013; 102(7):e293-e298

10 Manzoni P, Benjamin DK, Hope W, et al. The management of Candida infections in preterm neonates and the role of micafungin. J Matern Fetal Neonatal Med 2011;24 (Suppl 2):24-27

11 de la Torre P, Reboli AC. Micafungin: an evidence-based review of its place in therapy. Core Evid 2014;9:27-39

12 Chow BD, Linden JR, Bliss JM. Candida parapsilosis and the neonate: epidemiology, virulence and host defense in a unique patient setting. Expert Rev Anti Infect Ther 2012;10(8):935-946

13 Moudgal V, Little T, Boikov D, Vazquez JA. Multiechinocandin- and multiazole-resistant Candida parapsilosis isolates serially obtained during therapy for prosthetic valve endocarditis. Antimicrob Agents Chemother 2005;49(2):767-769

14 Monagle P, Chalmers E, Chan A, et al. Antithrombotic therapy in neonates and children: American College of Chest Physicians Evidence-Based Clinical Practice Guidelines (8th Edition). Chest 2008;133(6 Suppl):887S-968S
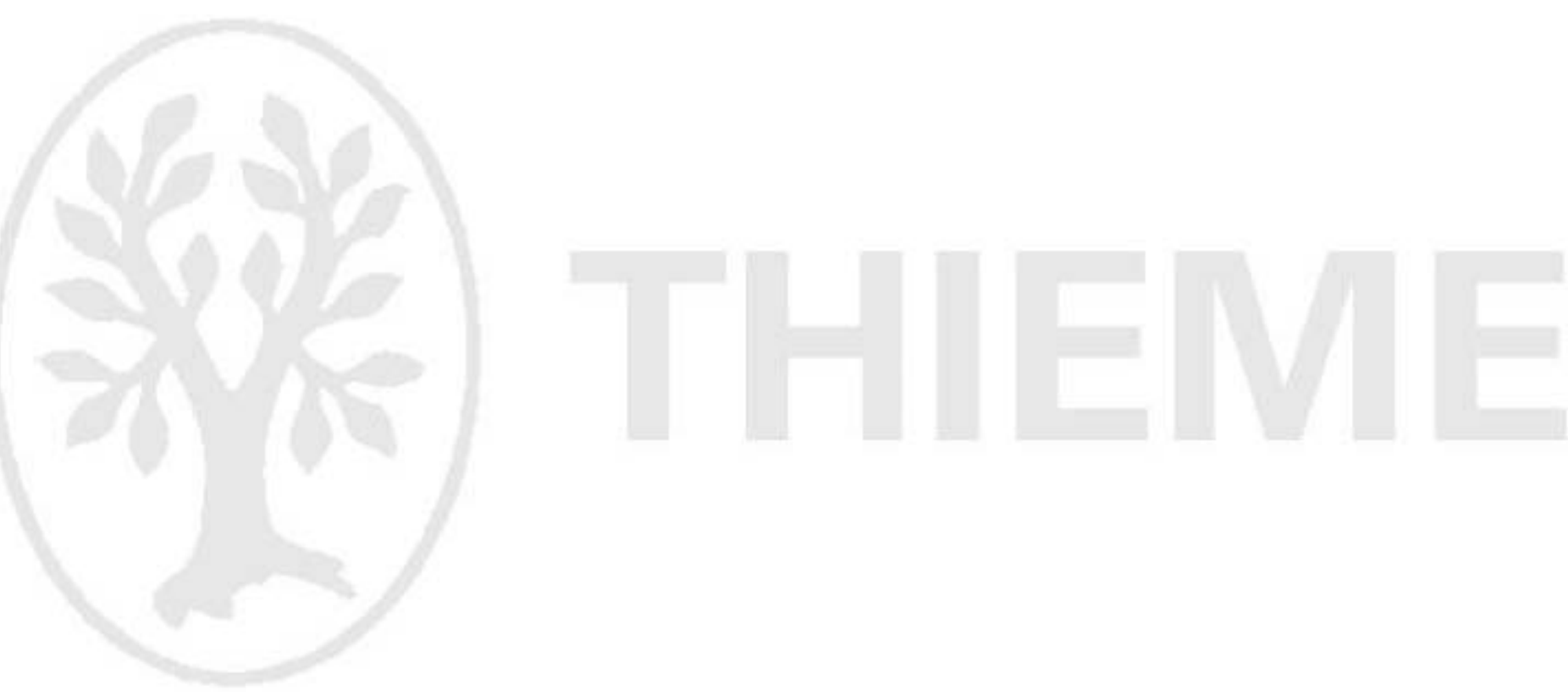